\title{
Atitudes alimentares e para com o ganho de peso e satisfação corporal de gestantes adolescentes
}

\author{
Eating attitudes, attitudes related to weight gain, \\ and body satisfaction of pregnant adolescents
}

Artigo Original

\section{Palavras-chave \\ Gravidez na adolescência Imagem corporal \\ Saúde do adolescente Comportamento do adolescente}

Keywords

Pregnancy in adolescence Body image Adolescent health Adolescent behavior

\section{Resumo}

OBJETIVO: Avaliar atitudes em relação à alimentação, ao ganho de peso e à imagem corporal de adolescentes grávidas. MÉTODOS: Adolescentes grávidas (n=67) foram avaliadas por meio do Questionário de Imagem Corporal, da Escala de Atitudes em relação ao Ganho de Peso na Gestação (AGPG) e de questões sobre comportamento de risco para transtornos alimentares e práticas não saudáveis para controle de peso. Associações entre as variáveis foram analisadas por meio dos testes ANOVA, Kruskal-Wallis, correlação de Pearson e correlação de Spearman. Uma regressão logística avaliou a influência das variáveis independentes com relação a omitir refeições, satisfação corporal e compulsão alimentar. RESULTADOS: As gestantes tinham, em média, 15,3 anos de idade (DP=1, 14) e 21,9 semanas de gestação (DP=6,53). O escore médio da AGPG foi 52,6 pontos, indicando boa atitude em relação ao ganho de peso, e 82, 1 \% das gestantes apresentaram satisfação corporal. As obesas apresentaram maior insatisfação corporal $(p=0,001)$ e as com sobrepeso pensavam mais em comida $(p=0,02)$ e em comer $(p=0,03)$. A frequência referida de compulsão alimentar foi $41,8 \%$, e, a de omitir refeições, 19\%. A regressão evidenciou que o Índice de Massa Corporal atual $(p=0,03 ; O R=1,18)$ e a importância da percepção do corpo/forma física antes da gestação $(p=0,03 ; O R=4,63)$ foram preditores para omitir refeições. Maior nível socioeconômico ( $p=0,04 ; O R=0,55)$ e maior preocupação com ganho de peso ( $p=0,04 ; O R=0,32)$ predisseram compulsão alimentar. CONCLUSÃO: Mesmo com a maioria das gestantes apresentando atitudes positivas em relação ao ganho de peso e satisfação corporal, as mais pesadas e mais preocupadas com ganho de peso tiveram maior risco de atitudes não saudáveis. As de menor classe social, menos preocupadas com ganho de peso e menos envergonhadas com seu corpo durante a gravidez, tiveram menor risco de atitudes não saudáveis.

\section{Abstract}

PURPOSE: To assess attitudes about eating, weight gain and body image of pregnant adolescents. METHODS: Pregnant adolescents (n=67) were assessed using the Body Image Questionnaire, the Attitude towards Weight Gain during Pregnancy scale (AWGP) and questions about risk behaviors for eating disorders and unhealthy weight control practices. Associations between variables were analyzed by ANOVA, Kruskal-Wallis test, Pearson and Spearman tests. The influence of the independent variables regarding skipping meals, body satisfaction and binge eating was evaluated by logistic regression. RESULTS: The average age of the adolescents was 15.3 years $(S D=1.14$ ) and their average gestational age was 21.9 weeks (SD=6.53). The average AWGP score was 52.6 points, indicating a positive attitude towards weight gain, and $82.1 \%$ of the pregnant girls were satisfied with their bodies. Obese girls had more body dissatisfaction ( $p=0.001$ ), and overweight girls thought more about food ( $p=0.02$ ) and eating ( $p=0.03$ ). The frequency of reported binge eating was $41.8 \%$, and the frequency of skipping meals was 19\%. Regression analysis showed that the current Body Mass Index ( $p=0.03 ; O R=1.18)$ and the importance of body awareness and fitness before pregnancy $(\mathrm{p}=0.03 ; \mathrm{OR}=4.63)$ were predictors of skipping meals. Higher socioeconomic level $(p=0.04 ; O R=0.55)$ and greater concern with weight gain $(p=0.03 ; O R=0.32)$ predicted binge eating. CONCLUSION: Even though the majority of the pregnant adolescents had positive attitudes toward weight gain and body satisfaction, those heavier and more concerned with weight gain had a higher risk of unhealthy attitudes, while those of lower social class, less concerned with weight gain and less embarrassed about their bodies during pregnancy, had a lower risk of unhealthy attitudes.
Correspondência

Carolina Oliboni

Departamento de Nutriç̃o da Faculdade de Saúde Pública da Universidade de São Paulo. Pós-graduacōono em Nutricão e Saúde Pública. Avenida Doutor Amaldo, $715,2^{\circ}$ andar - Pinheiros CEP: $01246-904$

São Paulo (SP), Brasi

Recebido

$18 / 08 / 2015$

Aceito com modificacōees $18 / 09 / 2015$
Clínica Obstétrica do Hospital das Clínicas da Faculdade de Medicina da Universidade de São Paulo - USP - São Paulo (SP), Brasil; Programa de Pós-Graduação em Nutrição em Saúde Pública do Departamento de Nutrição da Faculdade de Saúde Pública da Universidade de São Paulo - USP - São Paulo (SP), Brasil.

'Faculdade de Saúde Pública, Universidade de São Paulo - USP - São Paulo (SP), Brasil.

2Departamento de Nutrição, Faculdade de Saúde Pública, Universidade de São Paulo - USP - São Paulo (SP), Brasil. 


\section{Introdução}

As transformações ocorridas na adolescência podem levar o indivíduo a apresentar dificuldades em lidar com seu novo universo físico e mental, o que traz repercussões ao comportamento alimentar e à satisfação corporal ${ }^{1}$. A adolescência é uma fase de grande velocidade de crescimento, implicando uma necessidade aumentada de energia e nutrientes. Ser adolescente representa vivenciar mudanças físicas que acarretam alterações na aparência e no tamanho corporal, o que afeta diretamente imagem e satisfação corporais ${ }^{2}$.

A gestação também traz transformações físicas relacionadas aos ritmos metabólicos e hormonais e ao processo de integração de uma nova imagem, que exige da gestante uma resposta adaptativa ${ }^{3}$.

Se essas duas fases de intensas transformações ocorrerem ao mesmo tempo, podem representar para a adolescente um período difícil de compreensão, adaptação e aceitação das mudanças de compleição e de vida, podendo potencializar os riscos para o surgimento de problemas com a imagem e de comportamentos de risco para o desenvolvimento de transtornos alimentares (TA).

Atitudes alimentares disfuncionais, como omitir refeições, principalmente o café da manhã, substituir refeições como almoço e jantar por lanches, além da ingestão alta e frequente de refrigerantes e de alimentos com alta densidade energética, podem ser comuns na adolescência ${ }^{4}$. Fatores socioculturais exercem papel importante como desencadeantes de atitudes alimentares inadequadas $\mathrm{e}$ de insatisfação corporal em adolescentes, principalmente na sociedade ocidental, onde há obsessão por um corpo magro e perfeito, havendo valorização e exaltação, por parte da mídia, de celebridades com peso abaixo do esperado, enquanto a obesidade é estigmatizada ${ }^{5}$. Nessa fase há aumento de gordura corporal, levando não somente a mudanças na aparência física, mas também no próprio sentimento pelo corpo. Há um ideal de constituição física e, quanto mais ele distanciar-se do real, maior será a possibilidade de insatisfação com a imagem, o que pode desencadear atitudes alimentares inadequadas e transtornos alimentares ${ }^{6}$.

As transformações corporais da gravidez na adolescência ocorrem em um curto período, exigindo um processo de adaptação que certamente interfere na imagem corporal. O fato de o físico de uma gestante também não ser condizente com a imagem da adolescente valorizada pelos meios de comunicação ${ }^{7}$ pode acentuar a insatisfação. imagem corporal de gestantes deve ser alvo de atenção por parte dos profissionais, visando à promoção da saúde materna e infantil ${ }^{8}$.

Embora não seja formalmente reconhecida a ocorrência de TA na gestação, sugere-se que seja menos comum do que na população em geral ${ }^{9}$. Alguns estudos exploraram a presença desses transtornos nessa fase da vida, bem como a preocupação com o ganho de peso na gravidez ${ }^{10-13}$. A compulsão alimentar é o comportamento inadequado mais relatado durante o período gestacional, e geralmente é acompanhado de ganho de peso excessivo. Episódios compulsivos ocorrem com mais frequência em mulheres que costumam ter um padrão alimentar restritivo e não conseguem mantê-lo durante a gravidez ${ }^{12}$.

Mais ainda, o transtorno de compulsão alimentar (TCA) é relativamente comum na gestação, ocorrendo em 1 em cada 20 mulheres ${ }^{14}$. Estudos relatam frequência de TCA de 17 a $44 \%$ durante a gravidez ${ }^{12,15}$. Gestantes com TCA têm bebês com maior peso, grandes para a idade gestacional, e apresentam maior risco de parto cirúrgico - desfecho indiretamente influenciado pelo ganho excessivo de peso materno ${ }^{11}$. Tal ganho associado ao TCA durante a gestação representa maior risco de retenção de peso no pós-parto, obesidade, problemas com a imagem corporal e comprometimento da saúde da mãe e da criança ${ }^{13}$.

Devido ao fato de que muitas mulheres durante a gestação tentam controlar o peso com a adoção de métodos inadequados, é fundamental diagnosticar um TA na gravidez. Um TA gestacional pode levar a consequências negativas tanto para a mulher quanto para o feto, como altas porcentagens de abortos, baixo peso ao nascer, complicações obstétricas e depressão pós-parto ${ }^{9}$.

$\mathrm{Na}$ literatura brasileira, há uma lacuna de conhecimento acerca de problemas com a imagem corporal e atitudes alimentares inadequadas na gestação, especialmente entre adolescentes. Apenas um trabalho avaliou atitudes em relação ao peso de adolescentes gestantes, sem pesquisar satisfação corporal nem qualquer aspecto referente à alimentação nessa população ${ }^{7}$. Outro trabalho analisou satisfação corporal em gestantes, mas excluiu de sua pesquisa o termo "adolescentes", além de não ter incluído nenhum aspecto alimentar ${ }^{16}$. Recente revisão integrativa sobre insatisfação corporal em grávidas incluiu apenas adultas ${ }^{9}$ e encontrou um único trabalho nacional ${ }^{17}$, que examinou a relação entre força muscular do assoalho pélvico e variáveis de autopercepção corporal em mulheres grávidas.

Uma vez que a gestação na adolescência pode estar relacionada não apenas a problemas clínicos mas também a distúrbios da imagem corporal e comportamentos alimentares inadequados (incluindo comportamentos de risco para TA), a exploração desses temas em dois momentos de transformações concomitantes - a adolescência e a gestação - pode ser útil na prevenção e no tratamento de atitudes alimentares inadequadas, garantindo melhores condições de saúde para o crescimento adequado da adolescente e o desenvolvimento do feto. Assim, o objetivo deste 
estudo foi avaliar as atitudes em relação à alimentação, ao peso e ao corpo de um grupo de adolescentes grávidas.

\section{Métodos}

Este foi um estudo observacional transversal, com coleta de dados realizada no Programa Integrado de Assistência e Educação à Gestante Adolescente (PIAEGA) do ambulatório de Obstetrícia do Hospital das Clínicas da Faculdade de Medicina da Universidade de São Paulo (HCFMUSP).

Foram incluídas no estudo todas as gestantes adolescentes atendidas pelo PIAEGA entre abril e dezembro de 2013 que preencheram os critérios de inclusão:

- $\quad$ idade entre 11 e 17 anos;

- estar na faixa do $3^{\circ}$ ao $9^{\circ}$ mês de gestação $\left(10^{\mathrm{a}}\right.$ a $36^{\mathrm{a}}$ semana).

Foram consideradas inelegíveis as portadoras de algum transtorno psiquiátrico ou usuárias de drogas.

As adolescentes participantes preencheram a classificação socioeconômica segundo o Critério de Classificação Econômica Brasil (CCEB) da Associação Brasileira de Empresas de Pesquisa ${ }^{18}$. Foi também aplicada a Escala de Atitudes em relação ao Ganho de Peso na gestação (AGPG) em seu escore total e as seguintes subescalas previamente validadas:

1. Incômodo com o ganho de peso;

2. Preocupação com o peso;

3. Bem estar com a gravidez;

4. Ganharia mais peso se necessário ${ }^{19}$.

O Questionário de Imagem Corporal (BSQ) validado para adolescentes brasileiros ${ }^{20}$ apresenta questões específicas sobre classificação do peso ("muito abaixo", "abaixo do peso", "no peso certo", "acima ou muito acima do peso") e importância do mesmo ("não são muito importantes", "pesam um pouco em como eu me sinto", "são as coisas mais importantes que afetam como eu me sinto" e "eu me preocupo mais com a forma física do que com o peso") no período pré-gestacional e atual ${ }^{21}$. As adolescentes responderam ainda questões sobre comportamentos de risco para o desenvolvimento de TA (envolvendo restrição, compulsão, uso de laxantes e diuréticos ${ }^{22,23}$ e práticas não saudáveis para controle de peso (como comer muito pouco, tomar remédios para emagrecer, substituir alimentos e omitir refeições) adaptadas para o período gestacional ${ }^{21}$.

Dados sociodemográficos e características gestacionais foram obtidos por meio de perguntas diretas ou de coleta do cartão de atendimento das pacientes. As características antropométricas foram coletadas do prontuário das participantes para a classificação de adequação de peso gestacional, de acordo com o Institute of Medicine $(\mathrm{IOM})^{24}$.
Uma vez que a menarca ocorre, via de regra, no estágio M4 dos estágios de Tanner ${ }^{25}$ (ou seja, a penúltima fase do estadiamento puberal para meninas ${ }^{26}$ ) pode-se considerar que o crescimento das adolescentes que já engravidaram e que, portanto, já menstruaram, está praticamente em estágio final, podendo elas serem tomadas como adultas do ponto de vista fisiológico. Assim, a recomendação de ganho de peso gestacional do IOM $^{24}$ para adultas foi adequado para a população avaliada.

\section{Análises estatísticas}

A análise estatística dos dados foi realizada com o software SPSS 18.0 - Statistical Package for Social Sciences (SPSS, Chicago, Il, USA). Para todas as análises foi adotado um nível de significância de 5\%. Para avaliar a condição de normalidade foi aplicado o teste t-Student. As variáveis numéricas foram analisadas por meio do teste de Kruskal-Wallis.

Os cruzamentos de dados de variáveis numéricas que apresentavam distribuição normal foram analisados por meio da correlação de Pearson e, os não normais, pela correlação de Spearman. Os cruzamentos de variáveis numéricas com categóricas foram realizados pelo teste de Kruskal-Wallis, quando se comparou uma variável com vários grupos, e pelo de Mann-Whitney, quando se analisou uma variável em relação a dois grupos de variáveis somente (como no caso de trimestre gestacional, analisado como primeiro somado a segundo e terceiro). O teste de Mann-Whitney foi utilizado entre todos os pares para corrigir as análises múltiplas e, dessa forma, o valor para significância foi considerado quando $\mathrm{p}<0,008$.

As associações foram analisadas e diferenciadas em relação ao estado nutricional por meio do teste exato de Fisher.

Uma análise de regressão logística avaliou a influência das variáveis independentes com relação às variáveis dependentes (omitir refeição, satisfação corporal e compulsão alimentar). O procedimento de seleção das variáveis no modelo de regressão logística utilizado foi o Stepwise backwards, que inclui no modelo final as variáveis que, analisadas conjuntamente, apresentaram significância estatística $(\mathrm{p}<0,05)$. Foram apresentadas também as razões de chance (odds ratio) e o intervalo com $95 \%$ de confiança.

\section{Considerações Éticas}

O projeto foi aprovado pelo Comitê de Ética e Pesquisa da Faculdade de Saúde Pública da Universidade de São Paulo (USP) e pela Comissão de Ética para Análise de Projetos de Pesquisa do Hospital das Clínicas da Faculdade de Medicina da USP - Protocolo no 353/12. As participantes e um de seus pais assinaram um Termo de Consentimento Livre e Esclarecido após serem informados sobre os propósitos da pesquisa. 
Resultados

Foram incluídas 67 gestantes $(n=67)$ entre 12 e 17 anos, cujo perfil em relação à idade gestacional, IMC atual e pré-gestacional e idade da menarca encontram-se na Tabela 1 . Estavam no $1^{\circ}$ trimestre gestacional três gestantes $(4,5 \%)$, no $2^{\circ}$ trimestre, $43(64,2 \%)$ e, no $3^{\circ}$ trimestre, $21(31,3 \%)$ e portanto as do $1^{\circ}$ trimestre, em número muito reduzido em relação às demais, foram agrupadas às do $2^{\circ}$ trimestre. O IMC no momento da coleta de dados foi diferente entre os trimestres gestacionais, o que era esperado, como também o IMC atual das gestantes nos dois grupos (trimestres 1 e 2 e trimestre 3 ) era diverso.

No entanto, quando analisado pela classificação do estado nutricional, não houve diferença para a proporção de gestantes de diferentes trimestres $(\mathrm{p}=0,09)$. A maioria das gestantes, 38,8\% $(n=26)$, pertencia à classe socioeconômica $\mathrm{C} 1$, de acordo com o CCEB.

Quanto aos comportamentos de risco para TA ocorridos uma ou mais vezes por semana - portanto, comportamentos relevantes - $28(41,8 \%)$ referiram compulsão, $2(3 \%)$ disseram comer pouco e $1(1,5 \%)$ alegou fazer uso de laxante e diurético. Dentre as práticas não saudáveis para controle de peso, a mais frequente foi comer pouco $(11,9 \% ; n=8)$, seguida de omitir refeições $(28,4 \% ; n=19)$, prática marginalmente associada à idade $(\mathrm{p}=0,05)$. Ainda, três gestantes $(4,5 \%)$ referiram uso de substituto de refeição e uma $(1,5 \%)$ declarou consumir remédios para emagrecer. Não houve diferença entre trimestre e estado nutricional para comportamentos de risco de TA ou práticas não saudáveis para controle de peso.

Em relação à satisfação corporal, a média total no BSQ foi de 62,9 ( $\mathrm{DP}=28,2)$, sendo que o escore varia de 34 a 204 pontos e que, quanto maior a pontuação, pior é a insatisfação. A maioria das gestantes, não apresentou insatisfação corporal $(82,1 \%), 9 \%$ apresentou insatisfação leve, $6 \%$, moderada e, 3\%, grave. Quando o escore do BSQ foi analisado em função do estado nutricional atual, percebeu-se que aquelas com sobrepeso tinham maior insatisfação corporal comparado as de peso baixo e normal (Tabela 2). Não houve diferença na pontuação no BSQ entre os trimestres e a frequência de insatisfação corporal (nas classificações ausente, leve, moderada e grave) também não apresentou diferença $(\mathrm{p}=0,37)$.

No que se refere às atitudes em relação ao ganho de peso avaliadas pela AGPG, a média do escore da amostra geral foi de 52,6 (DP 10,5), sendo que o escore variava de 18 a 72 pontos e que, quanto maior a pontuação, mais positivas as atitudes. O escore da AGPG por trimestre gestacional e por classificação do estado nutricional pode ser visualizado na Tabela 2.

A análise comparativa do período de gestação com escores da AGPG evidenciou que gestantes do $1^{\circ}$ e $2^{\circ}$
Tabela 1. Características da amostra de gestantes adolescentes $(n=67$ ) de acordo com trimestre gestacional

\begin{tabular}{|c|c|c|c|c|}
\hline & $\begin{array}{c}\text { Amostra } \\
\text { total }\end{array}$ & $\begin{array}{l}1^{\circ} \text { e } 2^{\circ} \\
\text { trimestres }\end{array}$ & $3^{\circ}$ trimestre & Valor \\
\hline & $\begin{array}{l}\text { Média (DP); } \\
\text { mín-máx* }\end{array}$ & $\begin{array}{l}\text { Média (DP); } \\
\text { mín-máx }\end{array}$ & $\begin{array}{l}\text { Média (DP); } \\
\text { mín-máx }\end{array}$ & p \\
\hline Idade (anos) & $\begin{array}{c}15,3(1,1) \\
12-17\end{array}$ & $\begin{array}{c}15,3(1,1) \\
13-17\end{array}$ & $\begin{array}{c}15,4(1,3) \\
12-17\end{array}$ & 0,47 \\
\hline $\begin{array}{l}\text { Idade gestacional } \\
\text { (semanas) }\end{array}$ & $\begin{array}{l}21,9(6,5) \\
10-35\end{array}$ & $\begin{array}{c}18,2(3,4) \\
10-24\end{array}$ & $\begin{array}{l}30,0(3,8) \\
25-35\end{array}$ & 0,00 \\
\hline IMC atual $\left(\mathrm{kg} / \mathrm{m}^{2}\right)$ & $\begin{array}{c}23,9(4,6) ; \\
15,4-36\end{array}$ & $\begin{array}{c}23,0(4,3) ; \\
15,4-35\end{array}$ & $\begin{array}{l}26,0(3,7) ; \\
21,2-35,1\end{array}$ & 0,01 \\
\hline $\begin{array}{l}\text { IMC pré-gestacional } \\
\left(\mathrm{kg} / \mathrm{m}^{2}\right)\end{array}$ & $\begin{array}{c}21,2(3,8) ; \\
15-34,8\end{array}$ & $\begin{array}{l}20,8(3,8) \\
15,1-28,8\end{array}$ & $\begin{array}{l}22,2(3,6) \\
18,4-34,8\end{array}$ & 0,03 \\
\hline Idade menarca (anos) & $\begin{array}{l}11,8(1,4) \\
9-15\end{array}$ & $\begin{array}{l}11,8(1,2) \\
9-14\end{array}$ & $\begin{array}{l}11,8(1,7) \\
9-15\end{array}$ & 0,87 \\
\hline
\end{tabular}

DP: desvio padrão; mín: mínimo; , máx: máximo.

trimestres tiveram atitudes mais positivas do que as do $3^{\circ}$ trimestre apenas para a subescala 4 - "ganharia mais peso se necessário". Não houve diferença na pontuação da AGPG nas classificações do estado nutricional (Tabela 2).

Quanto à preocupação com peso e forma física antes e após o início da gestação, 50,7\% responderam que eram "nada ou pouco importantes" antes da gestação e após terem engravidado, esta resposta caiu para $44,8 \%$. Antes de engravidar, $49,3 \%$ consideravam o peso e a forma física "muito importantes" e após engravidarem esta resposta aumentou para 55,2\% (mas sem diferença entre estas duas avaliações $-\mathrm{p}=0,56$ ). Não houve diferença nesta resposta quanto ao trimestre gestacional na avaliação da preocupação antes $(\mathrm{p}=0,40)$ ou depois de engravidar $(\mathrm{p}=0,61)$.

Quanto à classificação sobre o próprio peso antes e após o início da gestação, a maioria referiu estar "no peso certo" antes de engravidar (58,2\%), 3,0\% classificava-se como "muito abaixo do peso", 22,4\%, "abaixo do peso", $14,9 \%$, "acima do peso" e 1,5\%, "muito acima do peso". Após engravidar, $3 \%$ ainda respondeu classificar-se como "muito abaixo do peso", 14,9\% como "abaixo do peso", $40 \%$ como "no peso certo", $35,8 \%$ como "acima do peso" e 6,0\% como "muito acima do peso". Quando comparadas àquelas que se viam como antes de engravidar, as do $1^{\circ}$ e $2^{\circ}$ trimestres gestacionais classificaram-se mais desta forma do que as do $3^{\circ}$ trimestre $(p=0,004)$.

A regressão logística avaliou a influência das variáveis independentes em relação à satisfação corporal (escore do BSQ), a omissão de refeição e a compulsão alimentar. Para essa análise, a importância do peso e da forma física foi agrupada em duas categorias: pouco ou nada importante ("não são muito importantes" e "pesam um pouco em como me sinto") e muito importantes ("são as coisas mais importantes que afetam como eu me sinto" e "eu me preocupo mais com a forma física do que com meu peso"). 
Tabela 2. Escore do Questionário de Imagem Corporal e da Escala de Atitudes em relação ao Ganho de Peso na Gestação total e fatores de gestantes adolescentes ( $\mathrm{n}=67$ )

\begin{tabular}{|c|c|c|c|c|c|c|c|c|}
\hline \multicolumn{9}{|c|}{ Total gestantes } \\
\hline & $\begin{array}{l}1^{\circ} \text { e } 2^{\circ} \\
\text { trimestres }\end{array}$ & $3^{\circ}$ trimestre & \multirow[t]{2}{*}{ Valor $p$} & Baixo peso & Peso normal & Sobrepeso & Obesidade & \multirow[t]{2}{*}{ Valor $p$} \\
\hline & média (DP) & média (DP) & & média (DP) & média (DP) & média (DP) & média (DP) & \\
\hline AGPG total & $54,9(7,6)$ & $47,6(14,0)$ & 0,09 & $60,0(6,1)$ & $53,9(10,7)$ & $48,7(11,1)$ & $51,7(6,7)$ & \\
\hline Fator 1 & $25,3(4,4)$ & $21,7(7,5)$ & 0,14 & $31,7(2,1)$ & $27,7(6,3)$ & $25,3(7,0)$ & $26,9(2,5)$ & \\
\hline Fator 2 & $14,0(2,5)$ & $13,1(3,2)$ & 0,43 & $10,0(2,0)$ & $9,1(2,3)$ & $8,7(2,5)$ & $10,1(2,1)$ & 0,21 \\
\hline BSQ total & $59,8(22,7)$ & $69,5(37,5)$ & 0,71 & $51,0(12,6)^{a}$ & $60,6(30,3)^{b}$ & $93,5(34,3)^{c}$ & $62,6(15,2)$ & $<0,001$ \\
\hline
\end{tabular}

adiferente de sobrepeso $(p<0,001)$; bdiferente de sobrepeso $(p=0,002)$; cdiferente do baixo peso $(p<0,001)$ e peso normal $(p=0,002)$. Fator 1 inclui oito questões $(3,4,9,12,13,14,16$ e 17), nomeado "incômodo com o ganho de peso"; Fator 2 inclui cinco questões $(1,2,5,8,15)$, nomeado "preocupação com o peso"; Fator 3 , nomeado "bem estar com a gravidez", inclui três questões (6,7 e 11); Fator 4, nomeado "ganharia mais peso se necessário", inclui duas questões (10 e 18).

Em relação à ocultar a refeição, quatro preditores foram encontrados:

- $\quad$ IMC atual maior ( $\mathrm{p}=0,03 ; \mathrm{OR}=1,18)$;

- $\quad$ percepção do peso e forma física antes da gravidez como "não importante" diminuem a chance de não comer em 78,3\%;

- concordância com a questão 4 da AGPG, ou seja, querer ganhar peso abaixo do recomendado na gestação, aumentou em $13 \%$ a chance de omitir refeição $(\mathrm{p}=0,04 ; \mathrm{OR}=13,08)$;

- concordância com a questão 9 da AGPG (ter menos vergonha do corpo) diminuiu em $97,9 \%$ a chance de omissão da alimentação $(\mathrm{p}=0,008 ; \mathrm{OR}=0,02)$.

Maior nível socioeconômico ( $\mathrm{p}=0,040 ; \mathrm{OD}=0,55)$ e maior preocupação com ganho de peso $(\mathrm{p}=0,037$; $\mathrm{OD}=0,32)$ predisseram compulsão alimentar.

Para a satisfação corporal, nenhuma das variáveis foi capaz de explicar o resultado avaliado pelo BSQ.

\section{Discussão}

Até onde se sabe, este foi o primeiro trabalho brasileiro a avaliar conjuntamente satisfação corporal, atitudes para com ganho de peso, comportamentos de risco para transtornos alimentares, e atitudes para com peso e alimentação entre gestantes adolescentes.

Em relação às práticas não saudáveis para controle de peso e comportamentos de risco para TA, a frequência foi menor do que a encontrada em estudos brasileiros com adolescentes não grávidas ${ }^{23,27}$. Entretanto, a frequência de compulsão alimentar foi mais alta do que a de outros estudos com adolescentes não grávidas e grávidas adultas: Leal $^{27}$ encontrou $11,9 \%$ e, Ferreira e Veiga ${ }^{23}, 37,7 \%$. Outros estudos encontraram frequências menores de compulsão alimentar em gestantes adultas: 17,3\% em bairros de baixa renda de duas cidades do Sul do Brasil ${ }^{12}, 19,2 \%$ também na mesma coorte do Sul do Brasil ${ }^{28}$ e $8,8 \%$ em Londres $(\mathrm{UK})^{29}$.
A prevalência de insatisfação corporal (18\% somando-se as categorias leve, moderada e grave) foi menor do que a maioria dos estudos com adolescentes do sexo feminino ${ }^{30-32}$. A gravidez parece ter se constituído um fator de proteção para insatisfação corporal nas adolescentes, sendo necessários outros estudos com população semelhante para que se confirme tal suposição.

A gestação pode representar uma janela de remissão dos sintomas do transtorno da compulsão alimentar para muitas mulheres, enquanto para outras representa um período de vulnerabilidade para o seu desenvolvimento ou agravamento, mesmo que os sintomas sejam reduzidos no decorrer da gravidez $z^{12,14,33}$.

Gestantes podem também fazer dieta para perda de peso até com 32 semanas de gestação e apresentar altas taxas de preocupação com o ganho de peso durante o terceiro trimestre de gestação ${ }^{14}$. O ganho de peso gestacional pode ser significativamente maior entre mulheres com compulsão alimentar durante a gravidez ${ }^{34}$.

O achado de maior frequência de compulsão alimentar no presente estudo, comparado a outros trabalhos com adolescentes ou gestantes, parece sugerir que gestação e adolescência, ocorrendo concomitantemente, podem representar pior predição para o desenvolvimento de compulsão. Novas avaliações com amostras de maior tamanho são necessárias para a confirmação dessa hipótese.

Deve-se, no entanto, considerar que a pergunta sobre compulsão alimentar pode não ter sido adequadamente interpretada, uma vez que o conceito explicitado no texto original do instrumento "comer uma quantidade de comida muito maior do que outra pessoa comeria" é subjetivo e, embora seguisse diretrizes do estudo de avaliação para aplicação em adolescentes no Brasil ${ }^{23}$, a pergunta pertencia a um questionário de autopreenchimento. E, obviamente, o resultado de $42 \%$ para compulsão alimentar nesta amostra não significa que essas gestantes tivessem um transtorno alimentar, especificamente.

Quanto às atitudes em relação ao ganho de peso na gestação, as adolescentes avaliadas apresentaram-se, em 
geral, positivas - resultado esse em concordância com os estudos que utilizaram a escala AGPG em gestantes adolescentes ${ }^{7,35,36}$. Porém, a análise para questões da AGPG que poderiam refletir piores atitudes indicou que gestantes mais pesadas afirmaram que não ganhariam $16 \mathrm{~kg}$ nem $18 \mathrm{~kg}$, mesmo se isso significasse que seus bebês seriam mais saudáveis. Os referidos resultados são semelhantes a outro estudo, no qual apesar de a maioria das adolescentes ter atitudes positivas em relação ao ganho de peso, aquelas com maiores pesos pré-gestacionais apresentaram atitudes mais negativas ${ }^{36}$, o que sugere que insatisfação corporal piora a postura em relação ao ganho de peso na gestação.

Desse modo, mesmo considerando que, teoricamente, adolescência e gestação são dois fatores que poderiam combinar-se para atitudes piores quanto ao corpo e peso, as adolescentes avaliadas, em sua maioria, não apresentavam insatisfação corporal e nem atitudes negativas em relação ao ganho de peso. Considera-se assim que a gestação pode ter se configurado em um fator protetor, já que um corpo maior representa o estado normal e adequado de uma gestação. Também a baixa renda da amostra pode ter constituído um outro fator protetor, uma vez que maior renda pode se relacionar à maior insatisfação corporal e atitudes inadequadas para com corpo e alimentação ${ }^{37}$.

Quando questionadas sobre ganhar mais peso do que o necessário (fator 4 da AGPG) se isso representasse que o bebê seria mais saudável, as gestantes dos $1^{\circ}$ e $2^{\circ}$ trimestres responderam mais positivamente do que as do $3^{\circ}$ trimestre, que então já não se mostraram receptivas a tal ganho de peso. De forma semelhante, as gestantes que se diziam "no peso certo" antes da gravidez mudaram sua percepção de peso após o início da mesma, passando a considerarem-se "acima do peso". Pode-se ponderar que as alterações no peso e na forma corporais típicas da gestação provavelmente fazem a mulher sentir-se maior ou gorda, mesmo com ganho de peso adequado, podendo ocorrer superestimação do tamanho corporal ${ }^{38}$ ou mudanças negativas na autoimagem, aumentando a insatisfação com o físico ainda que o peso antes da gravidez fosse normal ${ }^{39}$.

As gestantes adolescentes, em sua maioria, também afirmaram medo de ficarem gordas, mas deve-se considerar que, como as orientações de saúde focam massivamente na prevenção do ganho excessivo de peso durante a gestação, essa postura pode não representar uma atitude "negativa".

Como esse foi o primeiro estudo a utilizar a escala AGPG após adaptação transcultural, não é possível fazer comparações com outros estudos no Brasil. Recomendam-se novas avaliações com população gestante — adolescente e adulta - para avaliar diferentes aspectos em relação às modificações corporais e ganho de peso na gestação. De qualquer forma, os resultados são semelhantes ao estudo brasileiro que utilizou a versão anterior da AGPG com gestantes adultas em Goiânia-GO ${ }^{7}$, e a outros que encontraram, em geral, atitudes positivas em relação ao ganho de peso na gestação $0^{35,36}$, reforçando a ideia de preocupação com o bem estar do bebê e uma gestação saudável em detrimento da preocupação com corpo e forma física. Menor classe social significou menor risco de compulsão, assim como maior aceitação do ganho de peso. Isso aponta para importância da aceitação do ganho de peso e necessidade de avaliação de comportamentos inadequados na gestação entre diferentes extratos de renda, para uma melhor compreensão do valor do fator socioeconômico nesses achados.

Sobre omitir refeições, querer ganhar peso abaixo do recomendado na gestação aumentou a chance deste comportamento. Contudo, ter menos vergonha do corpo diminuiu essa ocorrência, resultado que novamente aponta para a importância de aceitação do ganho de peso da gestação e trabalho com a imagem corporal de gestantes. Dar maior valor ao corpo e à forma física antes da gestação também foi fator preditor para omitir refeições, chamando atenção para a avaliação da relação entre peso e imagem corporal na história de vida prévia da gestante, o que pode indicar certos comportamentos depois de grávida.

O pequeno tamanho amostral dessa pesquisa pode ter impedido a evidência de associações e dados mais condizentes com a literatura quanto à satisfação corporal e comportamentos alimentares entre adolescentes e grávidas. Por tratar-se de um estudo inédito em seus objetivos e população, não foi possível estabelecer o tamanho amostral exato para sua execução. A amostra incluiu todo o universo possível dentro do serviço especializado adotado como local de realização da pesquisa.

Deve-se considerar também que trabalhar com escalas de autopreenchimento envolve o risco de questões de caráter subjetivo, como a compulsão alimentar, não serem interpretadas de maneira exata. Uma exploração mais ampla só seria possível em um estudo qualitativo, de condução mais complexa na área da nutrição. Escalas e questionários são amplamente utilizados em pesquisas com imagem corporal e atitudes alimentares.

O presente estudo, de qualquer forma, traz resultados inéditos sobre um público ainda não explorado quanto aos construtos avaliados. A maioria das gestantes apresentou atitudes positivas em relação ao ganho de peso e satisfação corporal. No entanto, as mais pesadas e mais preocupadas com ganho de peso tiveram maior risco de atitudes não saudáveis, enquanto as de menor classe social, menos preocupadas com ganho de peso e menos envergonhadas sobre seu corpo atual, tiveram menor risco de atitudes não saudáveis. A compulsão alimentar foi o comportamento de risco para TA mais presente, e apresentou frequência mais alta do que a de outros trabalhos com adolescentes não grávidas e grávidas adultas. Mas, deve-se considerar 
a subjetividade da questão e possível superestimação deste resultado.

A relativamente alta frequência de compulsão alimentar sugere necessidade de avaliar-se o comportamento durante a gestação entre esse público nos serviços de saúde. Considerando-se que algumas adolescentes apresentaram maior risco de atitudes inadequadas, sugere-se também que os serviços de pré-natal atentem para aquelas mais preocupadas com seu corpo e peso e mais insatisfeitas com a imagem corporal, visando à prevenção de comportamentos inapropriados à saúde da gestante e do bebê.

\section{Agradecimentos}

A toda a equipe do PIAEGA, por autorizar e viabilizar esta pesquisa.

\section{Referências}

1. Conti MA, Frutuoso MF, Gambardela AM. Excesso de peso e insatisfação corporal em adolescentes. Rev Nutr. 2005;18(4):491-7.

2. Conti MA, Gambardella AM, Frutuoso MF. Insatisfação com a imagem corporal em adolescentes e sua relação com a maturação sexual. Rev Bras Crescimento Desenvolv Hum. $2005 ; 15(2): 36-44$

3. Araújo NM, Salim NR, Gualda DM, Silva LC. Corpo e sexualidade na gravidez. Rev Esc Enferm USP. 2012;46(3):552-8.

4. Leal GV, Philippi ST, Matsudo SM, Toassa EC. Consumo alimentar e padrão de refeições de adolescentes, São Paulo, Brasil. Rev Bras Epidemiol. 2010;13(3):457-67.

5. Cordás TA, Salzano FT. Aspectos gerais dos transtornos alimentares: características, critérios diagnósticos, epidemiologia e etiologia. In: Alvarenga M, Scagliusi FB, Philippi ST, organizadoras. Nutrição e transtornos alimentares: avaliação e tratamento. Barveri: Manole; 2010. p. 3-15

6. Carvalho RS, Amaral AC, Ferreira ME. Transtornos alimentares e imagem corporal na adolescência: uma análise da produção científica em psicologia. Psicol Teor Prat. 2009;1 1 (3):200-23.

7. Menezes IH, Domingues MH. Principais mudanças corporais percebidas por gestantes adolescentes assistidas em serviços públicos de saúde de Goiânia. Rev Nutr. 2004;17(2):185-94.

8. Meireles JF, Neves CM, Carvalho PH, Ferreira MC. Insatisfação corporal em gestantes: uma revisão integrativa da literatura. Ciênc Saúde Coletiva. 2015;20(7):2091-103.

9. Dunker KL, Alvarenga MS, Alves VP. Transtornos alimentares e gestação: uma revisão. J Bras Psiquiatr. 2009;58(1):60-8.

10. DiPietro JA, Millet S, Costigan KA, Gurewitsch E, Caulfield LE. Psychosocial influences on weight gain attitudes and behaviors during pregnancy. J Am Diet Assoc. 2003;103(10):1314-9.

11. Micali N, Treasure J, Simonoff E. Eating disorders symptoms in pregnancy: a longitudinal study of women with recent and past eating disorders and obesity. J Psychosom Res. 2007;63(3):297-303.

12. Soares RM, Nunes MA, Schmidt MI, Giacomello A, Manzolli P, Camey $S$, et al. Inappropriate eating behaviors during pregnancy: prevalence and associated factors among pregnant women attending primary care in southern Brazil. Int J Eat Disord. 2009;42(5):387-93.

13. Swann RA, Von Holle A, Torgersen L, Gendall K, Reichborn-Kjennerud T, Bulik CM. Attitudes toward weight gain during pregnancy: results from the Norwegian Mother and Child Cohort Study (MoBa). Int J Eat Disord. 2009;42(5):394-401.

14. Bulik CM, Von Holle A, Hamer R, Knoph Berg C, Torgersen L, Magnus $P$, et al. Patterns of remission, continuation and incidence of broadly defined eating disorders during early pregnancy in the Norwegian Mother and Child Cohort Study (MoBa). Psychol Med. 2007;37(8):1109-18.
15. Abraham S, King W, Llewellyn-Jones D. Attitudes to body weight, weight gain and eating behavior in pregnancy. J Psychosom Obstet Gynaecol. 1994;15(4):189-95.

16. Cairolli PB. Avaliação da imagem corporal e da (in)satisfação com o corpo grávido pela escala de medida em imagem corporal em gestantes inscritas no programa de pré-natal da rede básica de saúde de Vinhedo - SP [tese]. Campinas: Universidade Estadual de Campinas; 2009.

17. Sacomori C, Cardoso FL, Vanderlinde C. Pelvic floor muscle strength and body self perception among brazilian pregnant women. Physiotherapy. 2010;96(4):337-43.

18. Associação Brasileira de Empresas de Pesquisa [Internet]. Critério de Classificação Econômica Brasil. 2014 [citado 2014 Dez 12]. Disponível em: <http://www.abep.org/Servicos/Download. aspx?id $=01>$

19. Oliboni CM, Galletta MA, Francisco RP, Alvarenga MS. [Crosscultural adaptation of the pregnancy and weight gain attitude scale]. Rev Bras Ginecol Obstet. 2014;36(7):320-7. Portuguese.

20. Conti MA, Cordás TA, Latorre MR. A study of the validity and reliability of the Brazilian version of the Body Shape Questionnaire (BSQ) among adolescents. Rev Bras Saúde Matern Infant. 2009;9(3):331-8.

21. Neumark-Sztainer D, Story M, Resnick MD, Garwick A, Blum RW. Body dissatisfaction and unhealthy weight-control practices among adolescents with and without chronic illness: a population-based study. Arch Pediatr Adolesc Med. 1995; 149(12):1330-5.

22. Hay P. The epidemiology of eating disorder behaviors: an Australian community-based survey. Int J Eat Disord. 1998;23(4):371-82.

23. Ferreira JE, Veiga GV. Confiabilidade (teste-reteste) de um questionário simplificado para triagem de adolescentes com comportamentos de risco para transtornos alimentares em estudos epidemiológicos. Rev Bras Epidemiol. 2008; 1 1(3):393-401.

24. Institute of Medicine (US) and National Reasearch Council (US) Committee to Reexamine IOM Pregnancy Weight Guidelines; Rasmussen KM, Yaktine AL, editors. Weight gain during pregnancy: rexamining the guidelines. Washington (DC): National Academies Press; 2009.

25. Tanner JM. Growth at adolescence. Oxford: Blackwell; 1962.

26. Chipkevitch E. Avaliação clínica da maturação sexual na adolescência. J Pediatr (Rio J). 2001;77 Supl 2:S135-42.

27. Leal GV. Fatores associados ao comportamento de risco para transtornos alimentares em adolescentes na cidade de São Paulo [tese]. São Paulo: Universidade de São Paulo; 2013.

28. Nunes MA, Pinheiro AP, Hoffmann JF, Schmidt MI. Eating disorders symptoms in pregnancy and postpartum: a prospective study in a disadvantaged population in Brazil. Int J Eat Disord. 2014;47(4):426-30 
29. Easter A, Bye A, Taborelli E, Corfield F, Schmidt U, Treasure J, et al. Recognising the symptoms: how common are eating disorders in pregnancy? Eur Eat Disord Rev. 2013;21 (4):340-4.

30. Alves E, Vasconcelos FA, Calvo MC, Neves J. Prevalência de sintomas de anorexia nervosa e insatisfação com a imagem corporal em adolescentes do sexo feminino do Município de Florianópolis, Santa Catarina, Brasil. Cad Saúde Pública. 2008;24(3):503-12.

31. Martins CB, Pelegrini A, Matheus SC, Petroski EL. Insatisfação com a imagem corporal e relação com estado nutricional, adiposidade corporal e sintomas de anorexia e bulimia em adolescentes. Rev Psiquiatr Rio Gd Sul. 2010;32(1):19-23.

32. Beling MT. A auto imagem corporal e a tendência a transtornos alimentares em adolescentes do sexo feminino em Belo Horizonte, MG [tese]. Belo Horizonte: Universidade Federal de Minas Gerais; 2008

33. Knoph Berg C, Torgersen L, Von Holle A, Hamer RM, Bulik CM, Reichborn-Kjennerud T. Factors associated with binge eating disorder in pregnancy. Int J Eat Disord. $2011 ; 44(2)$ :124-33.
34. Nunes MA, Pinheiro AP, Camey SA, Schmidt MI. Binge eating during pregnancy and birth outcomes: a cohort study in a disadvantaged population in Brazil. Int J Eat Disord. 2012;45(7):827-31.

35. Stevens-Simon C, Nakashima I, Andrews D. Weight gain attitudes among pregnant adolescents. J Adolesc Health. 1993;14(5):369-72.

36. Gutierrez YM. Cultural factors affecting diet and pregnancy outcome of Mexican American adolescentes. J Adolesc Health. $1999 ; 25(3): 227-37$

37. Jeffery RW, French SA. Socioeconomic status and weight control practices among 20- to 45-year-old women. Am J Public Health. 1996;86(7): 1005-10.

38. Heinberg L, Guarda AS. Body image issues in obstetrics and gynecology. In Cash TF, Pruzinsky T, editors. Body image: a handbook of theory, research, and clinical practice. New York: Guilford; 2002. p. 351-60.

39. Fox $P$, Yamaguchi $C$. Body image change in pregnancy: a comparison of normal weight and overweight primigravidas. Birth. 1997;24(1):35-40. 